\title{
The Zahorski theorem is valid in Gevrey classes
}

\author{
by \\ Jean Sch mets (Liège) and Manuel Valdivia (Valencia)
}

\begin{abstract}
Let $\{\Omega, F, G\}$ be a partition of $\mathbb{R}^{n}$ such that $\Omega$ is open, $F$ is $F_{\sigma}$ and of the first category, and $G$ is $G_{\delta}$. We prove that, for every $\left.\gamma \in\right] 1, \infty[$, there is an element of the Gevrey class $\Gamma_{\gamma}$ which is analytic on $\Omega$, has $F$ as its set of defect points and has $G$ as its set of divergence points.
\end{abstract}

1. Introduction. Let $f$ be a real $\mathrm{C}_{\infty}$-function on $\mathbb{R}^{n}$. The set where $f$ is analytic is of course an open subset of $\mathbb{R}^{n}$; denote it by $\Omega_{f}$. It is clear that $x$ belongs to $\Omega_{f}$ if and only if the radius of convergence $\varrho_{f}(x)$ of the Taylor series of $f$ at $x$ is strictly positive and this series represents $f$ on some neighbourhood of $x$.

As the set $T_{f}$ of $x \in \mathbb{R}^{n}$ such that $\varrho_{f}(x)>0$ is easily seen to be an $F_{\sigma}$-set, the set $F_{f}=T_{f} \backslash \Omega_{f}$ is also $F_{\sigma}$ and its elements $x$ are characterized by the fact that $\varrho_{f}(x)>0$ and that the Taylor series of $f$ at $x$ represents $f$ on no neighbourhood of $x$. By use of a lemma of R. P. Boas ([1], p. 234), one easily sees that $F_{f}$ is also a first category set (cf. [3] or [4]).

Finally, one may consider the set $G_{f}=\mathbb{R}^{n} \backslash T_{f}$, a $G_{\delta}$-set given by $\varrho_{f}(x)=0$.

It is clear that $\left\{\Omega_{f}, G_{f}, F_{f}\right\}$ is a partition of $\mathbb{R}^{n}$.

The Zahorski theorem (cf. [6]) asserts conversely that for every partition $\{\Omega, F, G\}$ of $[0,1]$, where $\Omega$ is an open subset of $[0,1], F$ is a first category $F_{\sigma}$-subset of $[0,1]$ and $G$ is a $G_{\delta}$-subset of $[0,1]$, there is a real $\mathrm{C}_{\infty}$-function $f$ on $[0,1]$ such that $\Omega=\Omega_{f}, F=F_{f}$ and $G=G_{f}$. In [3], H. Salzmann and K. Zeller have provided a shorter proof of the Zahorski theorem and in [4], J. Siciak has extended this result to $\mathbb{R}^{n}$.

1991 Mathematics Subject Classification: Primary 26E10; Secondary 26 E05.

Key words and phrases: Gevrey classes, defect point, divergence point.

Research of the first author partially supported by the Belgian C.G.R.I.

Research of the second author partially supported by DGICYT PB91-0326 and the Convenio Hispano-Belga. 
The purpose of this paper is to prove that, for every $\gamma \in] 1, \infty[$, the Zahorski theorem has a solution $f$ belonging to the Gevrey class $\Gamma_{\gamma}$.

Let us recall that, for an open subset $\Omega$ of $\mathbb{R}^{n}$ and for $\left.\gamma \in\right] 1, \infty[$, the Gevrey class $\Gamma_{\gamma}(\Omega)$ is the set of $f \in \mathrm{C}_{\infty}(\Omega)$ for which there are constants $a, b>0$ such that

$$
\left\|\mathrm{D}^{\alpha} f\right\|_{\Omega} \leq a b^{|\alpha|}(|\alpha| !)^{\gamma}, \quad \forall \alpha \in \mathbb{N}_{0}^{n} .
$$

If $\Omega=\mathbb{R}^{n}$, we simply write $\Gamma_{\gamma}$ instead of $\Gamma_{\gamma}\left(\mathbb{R}^{n}\right)$.

It is known that

(a) a function $f \in \mathrm{C}_{\infty}(\Omega)$ belongs to $\Gamma_{\gamma}(\Omega)$ if and only if there are constants $c, d>0$ such that

$$
\left\|\mathrm{D}^{\alpha} f\right\|_{\Omega} \leq c d^{|\alpha|}|\alpha|^{\gamma|\alpha|}, \quad \forall \alpha \in \mathbb{N}_{0}^{n} .
$$

(b) the Denjoy-Carleman-Mandelbrojt result (cf. [2]) states that, for every closed ball $b$ of $\mathbb{R}^{n}$ and every $\left.\gamma \in\right] 1, \infty[$, there is a nonzero function $f \in \Gamma_{\gamma}$ with support contained in $b$.

In order to get an efficient way to state the results, for a real $\mathrm{C}_{\infty}$-function $f$ on $\mathbb{R}^{n}$, let us call the elements of $\Omega_{f}$ (resp. $F_{f} ; G_{f}$ ) the analytic points (resp. the defect points; the divergence points) of $f$.

The purpose of this article is to prove the following result.

TheOREM 1.1. For every partition $\{\Omega, F, G\}$ of $\mathbb{R}^{n}$, where $\Omega$ (resp. $F$; $G)$ is an open set (resp. a first category $F_{\sigma}$-set; a $G_{\delta}$-set) and every $\gamma \in$ ] $1, \infty\left[\right.$, there is an element of $\Gamma_{\gamma}$ having $\Omega($ resp. $F ; G)$ as its set of analytic points (resp. defect points; divergence points).

R e mark. It is a direct matter to check that the Zahorski theorem extends to the case when $\mathbb{R}^{n}$ is replaced by an open or a closed subset of $\mathbb{R}^{n}$.

2. An auxiliary result. We begin with the following easy result, where, as usual, $\mathrm{D}_{r}(\Omega)$ denotes the space of $\mathrm{C}_{r}$-functions on the open subset $\Omega$ of $\mathbb{R}^{n}$ which have a compact support contained in $\Omega$.

Proposition 2.1. Let $\Omega$ be a nonvoid open subset of $\mathbb{R}^{n}$. For every $f \in \mathrm{C}_{\infty}(\Omega), g \in \mathrm{D}_{\infty}(\Omega)$ and $\lambda>0$, it is well known that

$$
h(x)=\pi^{-n / 2} \lambda^{n} \int_{\Omega} f(y) g(y) e^{-\lambda^{2}|x-y|^{2}} d y
$$

belongs to $\mathrm{C}_{\infty}\left(\mathbb{R}^{n}\right)$. If moreover $a_{1}, a_{2}, b_{1}, b_{2}>0$ and $\zeta>1$ are such that

$$
\left\|\mathrm{D}^{\alpha} f\right\|_{\Omega} \leq a_{1} b_{1}^{|\alpha|}(|\alpha| !)^{\zeta} \quad \text { and } \quad\left\|\mathrm{D}^{\alpha} g\right\|_{\Omega} \leq a_{2} b_{2}^{|\alpha|}(|\alpha| !)^{\zeta}, \quad \forall \alpha \in \mathbb{N}_{0}^{n},
$$

then

$$
\left\|\mathrm{D}^{\alpha} h\right\|_{\mathbb{R}^{n}} \leq a_{1} a_{2}\left(b_{1}+b_{2}\right)^{|\alpha|}(|\alpha| !)^{\zeta}, \quad \forall \alpha \in \mathbb{N}_{0}^{n} .
$$


Proof. As $g$ has a compact support contained in $\Omega$, up to extension by 0 on $\mathbb{R}^{n} \backslash \Omega$, we may suppose that the product $f g$ is a $\mathrm{D}_{\infty}$-function on $\mathbb{R}^{n}$ with compact support contained in $\Omega$. So in the definition of $h(x)$, we may consider that we integrate on $\mathbb{R}^{n}$. Therefore integrating by parts $|\alpha|$ times gives

$$
\mathrm{D}^{\alpha} h(x)=\pi^{-n / 2} \lambda^{n} \int_{\mathbb{R}^{n}} \mathrm{D}^{\alpha}(f g)(y) e^{-\lambda^{2}|x-y|^{2}} d y,
$$

hence

$$
\begin{aligned}
\left|\mathrm{D}^{\alpha} h(x)\right| & \leq \pi^{-n / 2} \lambda^{n} \int_{\mathbb{R}^{n}} \sum_{\beta \leq \alpha}\left(\begin{array}{l}
\alpha \\
\beta
\end{array}\right)\left|\mathrm{D}^{\beta} f(y) \mathrm{D}^{\alpha-\beta} g(y)\right| e^{-\lambda^{2}|x-y|^{2}} d y \\
& \leq \sum_{\beta \leq \alpha}\left(\begin{array}{l}
\alpha \\
\beta
\end{array}\right) a_{1} a_{2} b_{1}^{|\beta|} b_{2}^{|\alpha-\beta|}(|\beta| !)^{\zeta}(|\alpha-\beta| !)^{\zeta} \\
& \leq a_{1} a_{2}\left(b_{1}+b_{2}\right)^{|\alpha|}(|\alpha| !)^{\zeta} .
\end{aligned}
$$

3. Special compact covers of open subsets of $\mathbb{R}^{n}$. In the following results, we are going to use systematically the following construction and notations. Let $\Omega$ be a nonvoid open subset of $\mathbb{R}^{n}$. Then we set

$$
\Omega_{m}=\left(\mathbb{R}^{n} \backslash \Omega\right) \cup\left\{x \in \mathbb{R}^{n}:|x| \geq m \sqrt{n}\right\}, \quad \forall m \in \mathbb{N},
$$

and denote by $\mu$ the first positive integer $m$ for which there is at least one cube $Q$ of the type

$$
\prod_{j=1}^{n}\left[2^{-m} a_{j}, 2^{-m}\left(a_{j}+1\right)\right] \quad \text { with } a \in \mathbb{Z}^{n}
$$

contained in $\Omega$ and such that $\mathrm{d}\left(Q, \Omega_{m}\right)>2^{-m} \sqrt{n}$. Let $Q_{1,1}, \ldots, Q_{1, p_{1}}$ be these cubes (of course, we have $p_{1} \in \mathbb{N}$ ) and set

$$
H_{1}=\bigcup_{h=1}^{p_{1}} Q_{1, h} .
$$

Now we proceed by recursion. If the sets $H_{1}, \ldots, H_{r}$ are obtained, we let $Q_{r+1,1}, \ldots, Q_{r+1, p_{r+1}}$ denote all the cubes of the type

$$
\prod_{j=1}^{n}\left[2^{-\mu-r} a_{j}, 2^{-\mu-r}\left(a_{j}+1\right)\right] \quad \text { with } a \in \mathbb{Z}^{n}
$$

contained in $\Omega$, disjoint from the interior of $H_{1} \cup \ldots \cup H_{r}$ and such that $\mathrm{d}\left(Q, \Omega_{\mu+r}\right)>2^{-\mu-r} \sqrt{n}$. Then we set

$$
H_{r+1}=\bigcup_{h=1}^{p_{r+1}} Q_{r+1, h}
$$


At this point let us remark that

$$
\mathrm{d}\left(H_{r}, H_{r+2}\right) \geq 2^{-\mu-r}, \quad \forall r \in \mathbb{N} .
$$

Finally, we set

$$
K_{r}=H_{1} \cup \ldots \cup H_{r}, \quad \forall r \in \mathbb{N} .
$$

It is clear that $\left\{K_{r}: r \in \mathbb{N}\right\}$ is a compact cover of $\Omega$ such that $K_{r} \subset K_{r+1}^{\mathrm{o}}$ for every $r \in \mathbb{N}$.

4. The auxiliary functions $v_{r}$. With this construction in mind and the notations therein, we now prove the following result.

Proposition 4.1. Let $\Omega$ be a nonvoid open subset of $\mathbb{R}^{n}$ and $\left.\zeta \in\right] 1, \infty[$. Then there are integers $c, d \in \mathbb{N}$ and functions $v_{r-2} \in \mathrm{C}_{\infty}\left(\mathbb{R}^{n}\right)$ for $r \in$ $\{3,4,5, \ldots\}$ such that
(a) $\operatorname{supp}\left(v_{r-2}\right) \subset K_{r+1} \backslash K_{r-2}^{\mathrm{o}}$,
(b) $v_{r-2}\left(\mathbb{R}^{n}\right) \subset[0,1]$,
(c) $v_{r-2}\left(H_{r}\right)=\{1\}$,
(d) $\left\|\mathrm{D}^{\alpha} v_{r-2}\right\|_{\mathbb{R}^{n}} \leq c\left(2^{r-2} d\right)^{|\alpha|}(|\alpha| !)^{\zeta}, \forall \alpha \in \mathbb{N}_{0}^{n}$,

for every integer $r>2$. that

Proof. Let $\varphi$ be an element of $\mathrm{C}_{\infty}(\mathbb{R})$ for which there are $l, d>0$ such

$$
\begin{gathered}
\varphi(t)>0 \quad \text { if }|t|<n^{-1 / 2} 2^{-\mu-4}, \\
\varphi(t)=0 \quad \text { if }|t| \geq n^{-1 / 2} 2^{-\mu-4}, \\
\left\|\varphi^{(s)}\right\|_{\mathbb{R}} \leq l d^{s}(s !)^{\zeta}, \quad \forall s \in \mathbb{N}_{0}, \quad \int_{\mathbb{R}} \varphi(t) d t=1 .
\end{gathered}
$$

The existence of such a $\varphi$ is provided by the Denjoy-Carleman-Mandelbrojt theorem (cf. [2]). Then we define

$$
\psi(x)=\varphi\left(x_{1}\right) \ldots \varphi\left(x_{n}\right), \quad \forall x \in \mathbb{R}^{n} .
$$

Clearly $\psi$ belongs to $\mathrm{C}_{\infty}\left(\mathbb{R}^{n}\right)$, has compact support equal to $\left[-n^{-1 / 2} 2^{-\mu-4}\right.$, $\left.n^{-1 / 2} 2^{-\mu-4}\right]^{n}$ and satisfies

$$
\left\|\mathrm{D}^{\alpha} \psi\right\|_{\mathbb{R}^{n}} \leq l^{n} d^{|\alpha|}(|\alpha| !)^{\zeta}, \quad \forall \alpha \in \mathbb{N}_{0}^{n}, \quad \int_{\mathbb{R}^{n}} \psi(x) d x=1 .
$$

Now for every integer $r \in \mathbb{N}$, we set

$$
\begin{aligned}
& L_{r}=\left\{x \in \mathbb{R}^{n}: \mathrm{d}\left(x, H_{r}\right) \leq 2^{-\mu-r-1}\right\}, \\
& \psi_{r}(x)=2^{(r-2) n} \psi\left(2^{r-2} x\right), \quad \forall x \in \mathbb{R}^{n},
\end{aligned}
$$


and note that $\psi_{r}$ belongs to $\mathrm{C}_{\infty}\left(\mathbb{R}^{n}\right)$ and has a compact support of diameter $2^{-\mu-r-1}$. Then we set

$$
v_{r-2}(x)=\psi_{r} * \chi_{L_{r}}(x)=\int_{\mathbb{R}^{n}} \psi_{r}(y) \chi_{L_{r}}(x-y) d y, \quad \forall x \in \mathbb{R}^{n} .
$$

It is well known or easy to check that the function $v_{r-2}$ belongs to $\mathrm{C}_{\infty}\left(\mathbb{R}^{n}\right)$ and has the properties (a)-(c) as well as

$$
\left\|\mathrm{D}^{\alpha} v_{r-2}\right\|_{\mathbb{R}^{n}} \leq \int_{\mathbb{R}^{n}}\left|\mathrm{D}^{\alpha} \psi_{r}\right| d x \leq \ell\left(\operatorname{supp}\left(\psi_{r}\right)\right)\left\|\mathrm{D}^{\alpha} \psi_{r}\right\|_{\mathbb{R}^{n}} \leq c\left(2^{r-2} d\right)^{|\alpha|}(|\alpha| !)^{\zeta}
$$

for every $\alpha \in \mathbb{N}_{0}^{n}$ if we set $c=l^{n}\left(n^{-1 / 2} 2^{-\mu-3}\right)^{n}$, a constant which does not depend on $r>2$ nor on $\alpha \in \mathbb{N}_{0}^{n}$.

5. Approximation in Gevrey classes and consequences. In the proof of Theorem 5.2, we shall make use of the following property which results immediately from the proof of Lemma 5 of [5].

Proposition 5.1 Let $r \in \mathbb{N}$ and $g \in \mathrm{D}_{r}\left(\mathbb{R}^{n}\right)$. Then, for every $\varepsilon>0$, there is $\lambda_{0}>0$ such that, for every $\lambda \geq \lambda_{0}$, the function

$$
h(x)=\pi^{-n / 2} \lambda^{n} \int_{\mathbb{R}^{n}} g(y) e^{-\lambda^{2}|x-y|^{2}} d y
$$

belongs to $\mathrm{C}_{\infty}\left(\mathbb{R}^{n}\right)$ (in fact, it is analytic on $\mathbb{R}^{n}$ ) and satisfies

$$
\left\|\mathrm{D}^{\alpha} h-\mathrm{D}^{\alpha} g\right\|_{\mathbb{R}^{n}} \leq \varepsilon \quad \text { if }|\alpha| \leq r .
$$

THEOREM 5.2. Let $\Omega$ be a nonvoid open subset of $\mathbb{R}^{n}$ and let $\zeta, \gamma$ be real numbers such that $1<\zeta<\gamma$. Then, for every $f \in \Gamma_{\zeta}(\Omega)$, there is $g \in \Gamma_{\gamma}(\Omega)$ which is analytic on $\Omega$ and such that

$$
\left\|\mathrm{D}^{\alpha} f-\mathrm{D}^{\alpha} g\right\|_{\Omega \backslash K_{s+1}} \leq \frac{1}{s} \quad \text { if }|\alpha| \leq s \text { and } s \geq 2,
$$

with $K_{s+1}$ defined as in the special compact cover of $\Omega$.

Proof. Of course there are numbers $a, b>1$ such that

$$
\left\|\mathrm{D}^{\alpha} f\right\|_{\Omega} \leq a b^{|\alpha|}(|\alpha| !)^{\zeta} \quad \text { and } \quad\left\|\mathrm{D}^{\alpha} v_{r-2}\right\|_{\Omega} \leq a\left(2^{r-2} b\right)^{|\alpha|}(|\alpha| !)^{\zeta}
$$

for every integer $r \geq 3$ and $\alpha \in \mathbb{N}_{0}^{n}$.

Now we introduce by recursion a sequence $\left(g_{s}\right)_{s \in \mathbb{N}}$ in $\mathrm{C}_{\infty}\left(\mathbb{R}^{n}\right)$ such that

$$
\left\|\mathrm{D}^{\alpha} g_{s}\right\|_{\mathbb{R}^{n}} \leq 2^{s-1} a^{s+1} 2^{(s+1)|\alpha|} b^{|\alpha|}(|\alpha| !)^{\zeta}, \quad \forall \alpha \in \mathbb{N}_{0}^{n}, \forall s \in \mathbb{N} .
$$

At this point, to get the functions $g_{s}$, we just need to consider a strictly increasing sequence $\left(\lambda_{s}\right)_{s \in \mathbb{N}}$ of $] 0, \infty[$ but later on we shall make more stringent restrictions on these positive numbers.

We start with

$$
g_{1}(x)=\pi^{-n / 2} \lambda_{1}^{n} \int_{\mathbb{R}^{n}} v_{1}(y) f(y) e^{-\lambda_{1}^{2}|x-y|^{2}} d y, \quad \forall x \in \mathbb{R}^{n},
$$


where of course $v_{1} f$ has been extended by 0 on $\mathbb{R}^{n} \backslash \Omega$. By Proposition 2.1, $g_{1}$ belongs to $\mathrm{C}_{\infty}\left(\mathbb{R}^{n}\right)$ and satisfies

$$
\left\|\mathrm{D}^{\alpha} g_{1}\right\|_{\mathbb{R}^{n}} \leq a^{2} 3^{|\alpha|} b^{|\alpha|}(|\alpha| !)^{\zeta} \leq 2^{0} a^{2} 2^{2|\alpha|} b^{|\alpha|}(|\alpha| !)^{\zeta}
$$

for every $\alpha \in \mathbb{N}_{0}^{n}$. Now if $g_{1}, \ldots, g_{s}$ are obtained, we first remark that we certainly have

$$
\begin{aligned}
\left\|\mathrm{D}^{\alpha}\left(f-\sum_{j=1}^{s} g_{j}\right)\right\|_{\Omega} & \leq\left\|\mathrm{D}^{\alpha} f\right\|_{\Omega}+\sum_{j=1}^{s}\left\|\mathrm{D}^{\alpha} g_{j}\right\|_{\Omega} \\
& \leq a b^{|\alpha|}(|\alpha| !)^{\zeta}+\sum_{j=1}^{s} 2^{j-1} a^{j+1} 2^{(j+1)|\alpha|} b^{|\alpha|}(|\alpha| !)^{\zeta} \\
& \leq 2^{s} a^{s+1} 2^{(s+1)|\alpha|} b^{|\alpha|}(|\alpha| !)^{\zeta}
\end{aligned}
$$

for every $\alpha \in \mathbb{N}_{0}^{n}$ and then check by direct use of Proposition 2.1 that the function $g_{s+1}$ defined by

$$
g_{s+1}(x)=\pi^{-n / 2} \lambda_{s+1}^{n} \int_{\mathbb{R}^{n}} v_{s+1}(y)\left(f(y)-\sum_{j=1}^{s} g_{j}(y)\right) e^{-\lambda_{s+1}^{2}|x-y|^{2}} d y
$$

suits our purpose.

Of course we have

$$
\begin{aligned}
\left\|\mathrm{D}^{\alpha} \sum_{j=1}^{s} g_{j}\right\|_{\mathbb{R}^{n}} & \leq \sum_{j=1}^{s} 2^{j-1} a^{j+1} 2^{(j+1)|\alpha|} b^{|\alpha|}(|\alpha| !)^{\zeta} \\
& \leq 2^{s} a^{s+1} 2^{(s+1)|\alpha|} b^{|\alpha|}(|\alpha| !)^{\zeta}
\end{aligned}
$$

for every $s \in \mathbb{N}$ and $\alpha \in \mathbb{N}_{0}^{n}$.

With this majorant at our disposal, we are in a position to make a more precise (but not yet final) choice of the numbers $\lambda_{s}$ (we are free to take them larger but strictly increasing). As we have

$$
\lim _{|\alpha| \rightarrow \infty} \frac{2^{s} a^{s+1} 2^{(s+1)|\alpha|} b^{|\alpha|}(|\alpha| !)^{\zeta}}{(|\alpha| !)^{\gamma}}=0, \quad \forall s \in \mathbb{N},
$$

there is a strictly increasing sequence $\left(A_{s}\right)_{s \in \mathbb{N}}$ in $\mathbb{N}$ such that, for every $s \in \mathbb{N}$,

$$
2^{s} a^{s+1} 2^{(s+1)|\alpha|} b^{|\alpha|}(|\alpha| !)^{\zeta} \leq(|\alpha| !)^{\gamma} \quad \text { if }|\alpha| \geq A_{s} .
$$

Then we can also fix a strictly increasing sequence $\left(B_{s}\right)_{s \in \mathbb{N}}$ in $\mathbb{N}$ such that

$$
\sup _{|\alpha| \leq A_{s}} a\left(2^{s} b\right)^{|\alpha|}(|\alpha| !)^{\zeta} \leq B_{s}, \quad \forall s \in \mathbb{N} .
$$

Next we introduce the following elements of $\mathrm{D}_{\infty}\left(\mathbb{R}^{n}\right)$ :

$$
h_{1}(x)= \begin{cases}v_{1}(x) f(x), & \forall x \in \Omega, \\ 0, & \forall x \in \mathbb{R}^{n} \backslash \Omega,\end{cases}
$$


and, for every integer $s \geq 2$,

$$
h_{s}(x)= \begin{cases}v_{s}(x)\left(f(x)-\sum_{j=1}^{s-1} g_{j}(x)\right), & \forall x \in \Omega, \\ 0, & \forall x \in \mathbb{R}^{n} \backslash \Omega .\end{cases}
$$

Finally, by recursive use of Proposition 5.1, we may require that the numbers $\lambda_{s}$ are such that, for every $s \in \mathbb{N}$,

$$
\left\|\mathrm{D}^{\alpha} h_{s}-\mathrm{D}^{\alpha} g_{s}\right\|_{\mathbb{R}^{n}} \leq\left(2^{s+2+A_{s+1}} B_{s+1}\right)^{-1} \quad \text { if }|\alpha| \leq A_{s+1} .
$$

Now we consider the series

$$
g(x)=\sum_{s=1}^{\infty} g_{s}(x), \quad \forall x \in \Omega .
$$

We first prove that $g$ is defined and belongs to $\mathrm{C}_{\infty}(\Omega)$. Indeed, for every $s \in \mathbb{N}$ and every $\alpha \in \mathbb{N}_{0}^{n}$ such that $|\alpha| \leq A_{s+1}$, we get

$$
\begin{aligned}
\left\|\mathrm{D}^{\alpha} h_{s+1}\right\|_{H_{s+2}} & \leq \sum_{\beta \leq \alpha}\left(\begin{array}{c}
\alpha \\
\beta
\end{array}\right)\left\|\mathrm{D}^{\beta} v_{s+1}\right\|_{H_{s+2}}\left\|\mathrm{D}^{\alpha-\beta}\left(f-\sum_{j=1}^{s} g_{j}\right)\right\|_{H_{s+2}} \\
& \leq \sum_{(*)}\left(\begin{array}{c}
\alpha \\
\beta
\end{array}\right) a\left(2^{s+1} b\right)^{|\beta|}(|\beta| !)^{\zeta}\left(2^{s+2+A_{s+1}} B_{s+1}\right)^{-1} \\
& \leq \sum_{\beta \leq \alpha}\left(\begin{array}{c}
\alpha \\
\beta
\end{array}\right) B_{s+1}\left(2^{s+2+A_{s+1}} B_{s+1}\right)^{-1} \\
& \leq 2^{-s-2-A_{s+1} 2^{|\alpha|} \leq 2^{-s-2}}
\end{aligned}
$$

(at $(*)$, we have used the fact that $f-\sum_{j=1}^{s} g_{j}=h_{s}-g_{s}$ on $H_{s+2}$ ). As $v_{s+1}(x)=0$ for every $x \in K_{s+1}$, we get

$$
\left\|\mathrm{D}^{\alpha} h_{s+1}\right\|_{K_{s+2}} \leq 2^{-s-2} \quad \text { if } s \in \mathbb{N} \text { and }|\alpha| \leq A_{s+1},
$$

hence

$$
\begin{aligned}
\left\|\mathrm{D}^{\alpha} g_{s+1}\right\|_{K_{s+2}} & \leq\left\|\mathrm{D}^{\alpha} g_{s+1}-\mathrm{D}^{\alpha} h_{s+1}\right\|_{\Omega}+\left\|\mathrm{D}^{\alpha} h_{s+1}\right\|_{K_{s+2}} \\
& \leq\left(2^{s+3+A_{s+2}} B_{s+2}\right)^{-1}+2^{-s-2} \leq 2^{-s-1}
\end{aligned}
$$

for every $s \in \mathbb{N}$ and $\alpha \in \mathbb{N}_{0}^{n}$ such that $|\alpha| \leq A_{s+1}$. Now it is clear that $g \in \mathrm{C}_{\infty}(\Omega)$.

We establish next that $g$ satisfies the inequalities announced in the statement of the theorem - in fact, we are going to prove more. Consider an integer $s \geq 2$ and a multi-index $\alpha \in \mathbb{N}_{0}^{n}$ such that $|\alpha| \leq A_{s}$-this is certainly the case if $|\alpha| \leq s$. For every $x_{0} \in \Omega \backslash K_{s+1}$, there is a first integer $p \geq 2$ such that $x_{0} \in K_{s+p}$. According to the last established inequality, we of course have

$$
\left|\mathrm{D}^{\alpha} g_{s+r}\left(x_{0}\right)\right| \leq 2^{-s-r}, \quad \forall r \in\{p-1, p, p+1, \ldots\}
$$


As $v_{s+p-2}\left(H_{s+p}\right)=\{1\}$, we also get

$$
\left|\mathrm{D}^{\alpha} f\left(x_{0}\right)-\mathrm{D}^{\alpha} \sum_{j=1}^{s+p-2} g_{j}\left(x_{0}\right)\right|=\left|\mathrm{D}^{\alpha} h_{s+p-2}\left(x_{0}\right)-\mathrm{D}^{\alpha} g_{s+p-2}\left(x_{0}\right)\right| \leq 2^{-s-p} \text {. }
$$

Thus

$$
\begin{aligned}
\left|\mathrm{D}^{\alpha} f\left(x_{0}\right)-\mathrm{D}^{\alpha} g\left(x_{0}\right)\right| & \leq\left|\mathrm{D}^{\alpha} f\left(x_{0}\right)-\mathrm{D}^{\alpha} \sum_{j=1}^{s+p-2} g_{j}\left(x_{0}\right)\right|+\sum_{r=p-1}^{\infty}\left|\mathrm{D}^{\alpha} g_{s+r}\left(x_{0}\right)\right| \\
& \leq 2^{-s-p}+\sum_{r=p-1}^{\infty} 2^{-s-r} \leq 2^{-s-p}+2^{-s-p+2}<\frac{1}{s},
\end{aligned}
$$

and hence

$$
\left\|\mathrm{D}^{\alpha} f-\mathrm{D}^{\alpha} g\right\|_{\Omega \backslash K_{s+1}} \leq \frac{1}{s} \quad \text { if } s \geq 2 \text { and }|\alpha| \leq A_{s} .
$$

At this step, if we proceed as in the proof of Lemma 6 of [5], we see that it is possible to select successively the numbers $\lambda_{s}$ in such a way that $g$ is an analytic function on $\Omega$. (This is our last refinement on the choice of $\lambda_{s}$.)

We still have to prove that $g \in \Gamma_{\gamma}(\Omega)$.

Set

$$
\sup _{|\alpha| \leq A_{2}}\left\|\mathrm{D}^{\alpha} g\right\|_{K_{3}} /(|\alpha| !)^{\gamma}=a_{1}
$$

and consider $\alpha \in \mathbb{N}_{0}^{n}$ and $x_{0} \in \Omega$.

On the one hand, if $|\alpha| \leq A_{2}$ and

(i) if $x_{0} \in K_{3}$, we trivially have

$$
\left|\mathrm{D}^{\alpha} g\left(x_{0}\right)\right| \leq\left\|\mathrm{D}^{\alpha} g\right\|_{K_{3}} \leq a_{1}(|\alpha| !)^{\gamma},
$$

(ii) if $x_{0} \in \Omega \backslash K_{3}$, we get

$$
\begin{aligned}
\left|\mathrm{D}^{\alpha} g\left(x_{0}\right)\right| & \leq\left|\mathrm{D}^{\alpha} f\left(x_{0}\right)\right|+\left|\mathrm{D}^{\alpha} f\left(x_{0}\right)-\mathrm{D}^{\alpha} g\left(x_{0}\right)\right| \\
& \leq a b^{|\alpha|}(|\alpha| !)^{\zeta}+\frac{1}{2} \leq 2 a b^{|\alpha|}(|\alpha| !)^{\gamma} .
\end{aligned}
$$

Hence there are constants $a_{2}, b_{2}>0$ such that

$$
\left\|\mathrm{D}^{\alpha} g\right\|_{\Omega} \leq a_{2} b_{2}^{|\alpha|}(|\alpha| !)^{\gamma} \quad \text { if }|\alpha| \leq A_{2} .
$$

On the other hand, if $|\alpha|>A_{2}$, we first let $s$ be the integer such that $A_{s}<|\alpha| \leq A_{s+1}$ (of course $s \geq 2$ ) and then consider the following two possibilities:

(i) if $x_{0} \in \Omega \backslash K_{s+2}$, then we have at once

$$
\begin{aligned}
\left|\mathrm{D}^{\alpha} g\left(x_{0}\right)\right| & \leq\left|\mathrm{D}^{\alpha} f\left(x_{0}\right)\right|+\left|\mathrm{D}^{\alpha} f\left(x_{0}\right)-\mathrm{D}^{\alpha} g\left(x_{0}\right)\right| \\
& \leq a b^{|\alpha|}(|\alpha| !)^{\zeta}+\frac{1}{s+1} \leq 2 a b^{|\alpha|}(|\alpha| !)^{\gamma},
\end{aligned}
$$


(ii) if $x_{0} \in K_{s+2}$, we have $\left|\mathrm{D}^{\alpha} g_{s+r}\left(x_{0}\right)\right| \leq 2^{-s-r}$ for every $r \in \mathbb{N}$, hence

$$
\sum_{r=1}^{\infty}\left|\mathrm{D}^{\alpha} g_{s+r}\left(x_{0}\right)\right| \leq \sum_{r=1}^{\infty} 2^{-s-r}=2^{-s} .
$$

But we also have

$$
\left|\mathrm{D}^{\beta} \sum_{j=1}^{s} g_{j}\left(x_{0}\right)\right| \leq 2^{s} a^{s+1} 2^{(s+1)|\beta|} b^{|\beta|}(|\beta| !)^{\zeta}
$$

for every $\beta \in \mathbb{N}_{0}^{n}$ and $s \in \mathbb{N}$, hence

$$
\left|\mathrm{D}^{\alpha} \sum_{j=1}^{s} g_{j}\left(x_{0}\right)\right| \leq(|\alpha| !)^{\gamma}
$$

since $|\alpha| \geq A_{s}$. Therefore

$$
\left|\mathrm{D}^{\alpha} g\left(x_{0}\right)\right| \leq\left|\mathrm{D}^{\alpha} \sum_{j=1}^{s} g_{j}\left(x_{0}\right)\right|+\sum_{r=1}^{\infty}\left|\mathrm{D}^{\alpha} g_{s+r}\left(x_{0}\right)\right| \leq 2^{-s}+(|\alpha| !)^{\gamma} \leq 2(|\alpha| !)^{\gamma} .
$$

Consequently, there are constants $a_{3}, b_{3}>0$ such that

$$
\left\|\mathrm{D}^{\alpha} g\right\|_{\Omega} \leq a_{3} b_{3}^{|\alpha|}(|\alpha| !)^{\gamma} \quad \text { if }|\alpha|>A_{2}
$$

COROLlary 5.3. For every open and nonvoid subset $\Omega$ of $\mathbb{R}^{n}$ and $\gamma \in$ ] $1, \infty\left[\right.$, there is a function $g \in \Gamma_{\gamma}$ which is

(a) analytic on $\Omega$,

(b) identically 0 on no connected component of $\Omega$,

(c) flat on $\mathbb{R}^{n} \backslash \Omega$ (i.e. identically 0 together with all its derivatives on $\left.\mathbb{R}^{n} \backslash \Omega\right)$.

Proof. If $\Omega$ is connected, we choose $\zeta \in] 1, \gamma\left[\right.$ and $f \in \Gamma_{\zeta}(\Omega)$ with compact support contained in $K_{4}^{\mathrm{o}} \backslash K_{3}$ and such that $\|f\|_{\Omega}>1 / 2$. Then Theorem 5.2 provides $g \in \Gamma_{\gamma}(\Omega)$ which is analytic on $\Omega$ and such that

$$
\left\|\mathrm{D}^{\alpha} f-\mathrm{D}^{\alpha} g\right\|_{\Omega \backslash K_{2+1}} \leq \frac{1}{2} \quad \text { if }|\alpha| \leq 2
$$

(which implies that $g$ is not identically 0 on $\Omega$ ) as well as

$$
\left\|\mathrm{D}^{\alpha} g\right\|_{\Omega \backslash K_{s+1}}=\left\|\mathrm{D}^{\alpha} g-\mathrm{D}^{\alpha} f\right\|_{\Omega \backslash K_{s+1}} \leq \frac{1}{s} \quad \text { if }|\alpha| \leq s \text { and } s \geq 3 .
$$

It is then well known that extending $g$ by 0 on $\mathbb{R}^{n} \backslash \Omega$ provides a solution.

If $\Omega$ has a finite number of connected components - say $\Omega_{1}, \ldots, \Omega_{m}$ then, for every $k \in\{1, \ldots, m\}$, there is $g_{k} \in \Gamma_{\gamma}$ which is analytic on $\Omega_{k}$, not identically 0 on $\Omega_{k}$ and flat on $\mathbb{R}^{n} \backslash \Omega_{k}$. It is then clear that $g=\sum_{k=1}^{m} g_{k}$ is a solution.

As $\Omega$ always has countably many connected components, to conclude, we just have to settle the case when $\left\{\Omega_{m}: m \in \mathbb{N}\right\}$ is the set of connected 
components of $\Omega$. For this purpose, fix $\zeta \in] 1, \gamma[$. By the first part of the proof, for every $m \in \mathbb{N}$, there is $g_{m} \in \Gamma_{\zeta}$ which is analytic on $\Omega_{m}$, not identically 0 on $\Omega_{m}$, flat on $\mathbb{R}^{n} \backslash \Omega_{m}$ and such that

$$
\left\|\mathrm{D}^{\alpha} g_{m}\right\|_{\Omega_{m} \backslash K_{m, s+1}} \leq \frac{1}{s} \quad \text { if }|\alpha| \leq s \text { and } s \geq 2,
$$

where of course $K_{m, r}$ is the $r$ th element of the special cover of $\Omega_{m}$. So, for every $m \in \mathbb{N}$, there are constants $a_{m}, b_{m}>1$ such that

$$
\left\|\mathrm{D}^{\alpha} g_{m}\right\|_{\mathbb{R}^{n}} \leq a_{m} b_{m}^{|\alpha|}(|\alpha| !)^{\zeta}, \quad \forall \alpha \in \mathbb{N}_{0}^{n},
$$

hence there is an integer $k_{m} \geq m$ such that

$$
a_{m} b_{m}^{|\alpha|}(|\alpha| !)^{\zeta} \leq(|\alpha| !)^{\gamma} \quad \text { if }|\alpha| \geq k_{m} .
$$

Then we set

$$
c_{m}=\left(\sup _{|\alpha| \leq k_{m}} a_{m} b_{m}^{|\alpha|}(|\alpha| !)^{\zeta}\right)^{-1} \quad \text { and } \quad g=\sum_{m=1}^{\infty} 2^{-m} c_{m} g_{m} .
$$

It is clear that $g$ is a function defined on $\mathbb{R}^{n}$ which is analytic on $\Omega$, identically 0 on no connected component of $\Omega$ and identically 0 on $\mathbb{R}^{n} \backslash \Omega$. Now we prove that $g$ belongs to $\mathrm{C}_{\infty}\left(\mathbb{R}^{n}\right)$ and is flat on $\mathbb{R}^{n} \backslash \Omega$. Let $x \in \mathbb{R}^{n} \backslash \Omega$. For every integer $k \geq 3$, there is $r>0$ such that the ball $b=\left\{y \in \mathbb{R}^{n}:|x-y| \leq r\right\}$ is disjoint from the compact sets $K_{1, k+1}, \ldots, K_{k, k+1}$. For every $\alpha \in \mathbb{N}_{0}^{n}$ such that $|\alpha| \leq k$, this leads to

$$
\begin{aligned}
& \sup _{x \in b} \sum_{m=1}^{\infty} 2^{-m} c_{m}\left|\mathrm{D}^{\alpha} g_{m}\right| \\
& \quad \leq \sup \left\{\sup _{m \leq k} \frac{2^{-m} c_{m}}{k}, \sup _{m>k} 2^{-m} c_{m}\left\|\mathrm{D}^{\alpha} g_{m}\right\|_{\mathbb{R}^{n}}\right\} \leq \sup \left\{1 / k, 2^{-k}\right\},
\end{aligned}
$$

hence $g$ belongs to $\mathrm{C}_{\infty}\left(\mathbb{R}^{n}\right)$ and is flat on $\mathbb{R}^{n} \backslash \Omega$. We still have to prove that $g \in \Gamma_{\gamma}$. This is immediate: for every $\alpha \in \mathbb{N}_{0}^{n}$, we have

$$
\left\|\mathrm{D}^{\alpha} g\right\|_{\mathbb{R}^{n}}=\sup _{m \in \mathbb{N}} 2^{-m} c_{m}\left\|\mathrm{D}^{\alpha} g_{m}\right\|_{\mathbb{R}^{n}} \leq \sup _{m \in \mathbb{N}} \sup \left\{2^{-m}, 2^{-m}(|\alpha| !)^{\gamma}\right\} \leq(|\alpha| !)^{\gamma}
$$

by consideration of the cases $|\alpha| \leq k_{m}$ and $|\alpha|>k_{m}$.

COROllary 5.4. For every $\gamma \in] 1, \infty[$ and nondegenerate compact intervals $I, J$ of $\mathbb{R}$ such that $J \subset I^{\circ}$, there are $f \in \mathrm{C}_{\infty}(\mathbb{R})$ and $c>0$ such that

(a) $f$ has no divergence point,

(b) $f(\mathbb{R}) \subset[0,1]$,

(c) $\left.\left.f(\mathbb{R} \backslash I)=\{0\}, f\left(I^{\mathrm{o}}\right) \subset\right] 0,1\right]$ and $f(J)=\{1\}$,

(d) $\left\|f^{(k)}\right\|_{\mathbb{R}} \leq c k^{\gamma k}, \forall k \in \mathbb{N}$. 
Proof. Let $I=\left[a_{1}, b_{1}\right]$ and $J=\left[a_{2}, b_{2}\right]$. We choose $\left.\zeta \in\right] 1, \gamma[$ and apply Corollary 5.3 with $\Omega=] a_{1}, a_{2}\left[\right.$ : there is a nonzero $g \in \Gamma_{\zeta}$ which is analytic on $] a_{1}, a_{2}\left[\right.$ and such that $\operatorname{supp}(g)=\left[a_{1}, a_{2}\right]$. Now we choose $k>0$ such that $g_{1}=k g^{2}$ satisfies $\int_{\mathbb{R}} g_{1}(x) d x=1$ and define the function $f_{1}$ on $\mathbb{R}$ by

$$
f_{1}(x)=\int_{-\infty}^{x} g_{1}(t) d t, \quad \forall x \in \mathbb{R} .
$$

It is clear that $f_{1}$ belongs to $\Gamma_{\zeta}$, is analytic on $] a_{1}, a_{2}$ [ and satisfies

$$
\left.\left.\left.\left.f_{1}(]-\infty, a_{1}\right]\right)=\{0\}, \quad f_{1}(] a_{1}, a_{2}[) \subset\right] 0,1\right] \quad \text { and } \quad f_{1}\left(\left[a_{2}, \infty[)=\{1\} .\right.\right.
$$

So it is clear that $f_{1}$ has no divergence point.

Similarly there is $f_{2} \in \Gamma_{\zeta}$ which is analytic on $] b_{2}, b_{1}[$, has no divergence point and satisfies

$$
\left.\left.\left.\left.f_{2}(]-\infty, b_{2}\right]\right)=\{0\}, \quad f_{2}(] b_{2}, b_{1}[) \subset\right] 0,1\right] \quad \text { and } \quad f_{2}\left(\left[b_{1}, \infty[)=\{1\} .\right.\right.
$$

Finally, we set

$$
f(x)=f_{1}(x) f_{2}\left(b_{1}+b_{2}-x\right), \quad \forall x \in \mathbb{R} .
$$

Of course $f$ belongs to $\Gamma_{\zeta}$ and satisfies (a)-(c). Let us establish that $f$ also satisfies (d). As $f \in \Gamma_{\zeta}$, there are $a, b>0$ such that

$$
\left\|f^{(k)}\right\|_{\mathbb{R}} \leq a b^{k} k^{\zeta k}, \quad \forall k \in \mathbb{N}_{0} .
$$

Since

$$
\lim _{k \rightarrow \infty} \frac{a b^{k} k^{\zeta k}}{k^{\gamma k}}=0
$$

there is $k_{0} \in \mathbb{N}$ such that $a b^{k} k^{\zeta k} \leq k^{\gamma k}$ for every $k \geq k_{0}$; therefore there is $c>0$ such that

$$
\left\|f^{(k)}\right\|_{\mathbb{R}} \leq c k^{\gamma k}, \quad \forall k \in \mathbb{N} .
$$

Proposition 5.5. Let $\gamma \in] 1, \infty[$, let $p \in \mathbb{N}$ and let $I, J$ be nondegenerate compact intervals of $\mathbb{R}$ such that $J \subset I^{\mathrm{O}}$. Then there is $m_{0} \in \mathbb{N}$ such that, for every integer $m \geq m_{0}$, there is a function $u \in \mathrm{C}_{\infty}(\mathbb{R})$ satisfying the following conditions:

(a) $u$ has no divergence point,

(b) $\operatorname{supp}(u) \subset I$,

(c) $\left\|u^{(k)}\right\|_{\mathbb{R}} \leq 2^{-m}, \forall k \in\{0,1, \ldots, m\}$,

(d) $\left\|u^{(k)}\right\|_{\mathbb{R}} \leq 2^{k} k^{\gamma k}, \forall k \in \mathbb{N}$,

(e) for every $x \in J$, one has either

$$
\left|u^{(p m)}(x)\right| \geq 5^{-m} m^{\gamma(p-1) m}
$$

or

$$
\left|u^{(p m+1)}(x)\right| \geq 5^{-(m+1 / p)}\left(\frac{p m+1}{p+1}\right)^{\gamma(p-1)(m+1 / p)} .
$$


Proof. Corollary 5.4 provides a function $f \in \mathrm{C}_{\infty}(\mathbb{R})$ and a constant $c>0$ such that $f$ has no divergence point and satisfies

$$
f(\mathbb{R}) \subset[0, c], \quad f(\mathbb{R} \backslash I)=\{0\}, \quad f(J)=\{1\}
$$

as well as

$$
\left\|f^{(k)}\right\|_{\mathbb{R}} \leq c k^{\gamma k}, \quad \forall k \in \mathbb{N} .
$$

For any $m \in \mathbb{N}$, we can introduce

$$
\begin{aligned}
a & =m^{\gamma}, \quad b=\left(2^{2 m} c^{\gamma m}\right)^{-1}, \\
u(x) & =b f(x) \sin (a x), \quad \forall x \in \mathbb{R} .
\end{aligned}
$$

It is then clear that $u$ is a $\mathrm{C}_{\infty}$-function on $\mathbb{R}$ satisfying the conditions (a) and (b) as well as

$$
\|u\|_{\mathbb{R}} \leq b c=\left(2^{2 m} m^{\gamma m}\right)^{-1} \leq 2^{-m} .
$$

Moreover, for every $x \in \mathbb{R}$ and $k \in \mathbb{N}$, we have

$$
\begin{aligned}
\left|u^{(k)}(x)\right| & \leq b \sum_{h=0}^{k}\left(\begin{array}{l}
k \\
h
\end{array}\right)\left|f^{(h)}(x)\right| a^{k-h} \\
& \leq b c \sum_{h=0}^{k}\left(\begin{array}{l}
k \\
h
\end{array}\right) h^{\gamma h} a^{k-h} \leq\left(2^{2 m} m^{\gamma m}\right)^{-1}\left(k^{\gamma}+a\right)^{k},
\end{aligned}
$$

hence

$$
\left|u^{(k)}(x)\right| \leq\left(2^{2 m} m^{\gamma m}\right)^{-1}\left(m^{\gamma}+m^{\gamma}\right)^{m}=2^{-m} \quad \text { if } 1 \leq k \leq m
$$

as well as

$$
\left|u^{(k)}(x)\right| \leq\left(2^{2 m} m^{\gamma m}\right)^{-1}\left(k^{\gamma}+k^{\gamma}\right)^{k} \leq 2^{k} k^{\gamma k} \quad \text { if } k>m ;
$$

i.e. $u$ also satisfies the conditions (c) and (d).

Now we investigate (e). Let $x \in J$. Of course we have

$$
u^{(k)}(x)=a^{k} b \sin (k \pi / 2+a x), \quad \forall k \in \mathbb{N} .
$$

Now, for every $m \in \mathbb{N}$, we certainly have

$$
\sup \{|\sin (p m \pi / 2+a x)|,|\sin ((p m+1) \pi / 2+a x)|\} \geq 2^{-1 / 2} .
$$

So on the one hand, if $|\sin (p m \pi / 2+a x)| \geq 2^{-1 / 2}$, we get

$$
\left|u^{(p m)}(x)\right| \geq 2^{-1 / 2} a^{p m} b=4^{-m}(\sqrt{2} c)^{-1} m^{\gamma(p-1) m},
$$

and on the other hand, if $|\sin ((p m+1) \pi / 2+a x)| \geq 2^{-1 / 2}$, then

$$
\begin{aligned}
\left|u^{(p m+1)}(x)\right| & \geq 2^{-1 / 2} a^{p m+1} b=4^{-m}(\sqrt{2} c)^{-1} m^{\gamma((p-1) m+1)} \\
& \geq 4^{-(m+1 / p)}(\sqrt{2} c)^{-1} m^{\gamma(p-1)(m+1 / p)} \\
& \geq 4^{-(m+1 / p)}(\sqrt{2} c)^{-1}\left(\frac{p m+1}{p+1}\right)^{\gamma(p-1)(m+1 / p)} .
\end{aligned}
$$


To conclude, it is enough to take as $m_{0}$ any positive integer $m_{0}$ such that

$$
\sup \left\{4(\sqrt{2} c)^{1 / m_{0}}, 4(\sqrt{2} c)^{1 /\left(m_{0}+1 / p\right)}\right\} \leq 5 .
$$

6. Characterizing the sets of divergence points. In this section, we establish the following result.

Theorem 6.1. For every $\gamma \in] 1, \infty\left[\right.$ and every $G_{\delta}$-subset $G$ of $\mathbb{R}^{n}$, there is an element of $\Gamma_{\gamma}$ having $G$ as its set of divergence points.

Proof. We proceed in several steps.

Step 1: the numbers $\gamma_{j}$ and $p_{j}$. We fix a strictly increasing sequence $\left.\left(\gamma_{j}\right)_{j \in \mathbb{N}_{0}} \subset\right] 1, \gamma\left[\right.$ and, for every $j \in \mathbb{N}$, denote by $p_{j}$ a positive integer such that $p_{j}\left(\gamma_{j}-\gamma_{j-1}\right)>\gamma_{j}$.

Step 2: some auxiliary inequalities and the numbers $q_{r}$. For every $r \in$ $\mathbb{N}$, we certainly have

$$
\frac{p_{r}-1}{p_{r}} \gamma_{r}>\gamma_{r-1}>\ldots>\gamma_{0}>1 .
$$

Therefore, for every $j \in\{0, \ldots, r-1\}$, it is a straightforward matter to check the following limits:

$$
\begin{array}{r}
\lim _{m \rightarrow \infty} 5^{m} 2^{2 p_{r} m}\left(p_{r} m\right)^{\gamma_{j} p_{r} m} \frac{1}{m^{\gamma_{r}\left(p_{r}-1\right) m}} \\
=\lim _{m \rightarrow \infty}\left(\frac{4.5^{1 / p_{r}} p_{r}^{\gamma_{j}}}{m^{\gamma_{r}\left(p_{r}-1\right) / p_{r}-\gamma_{j}}}\right)^{p_{r} m}=0, \\
\lim _{m \rightarrow \infty} 5^{m+1 / p_{r}} 2^{2\left(p_{r} m+1\right)}\left(p_{r} m+1\right)^{\gamma_{j}\left(p_{r} m+1\right)}\left(\frac{p_{r}+1}{p_{r} m+1}\right)^{\gamma_{r}\left(p_{r}-1\right)\left(m+1 / p_{r}\right)} \\
=\lim _{m \rightarrow \infty}\left(\frac{4.5^{1 / p_{r}}\left(p_{r}+1\right)^{\gamma_{r}\left(p_{r}-1\right) / p_{r}}}{\left(p_{r} m+1\right)^{\gamma_{r}\left(p_{r}-1\right) / p_{r}-\gamma_{j}}}\right)^{p_{r} m+1}=0,
\end{array}
$$

$\lim _{m \rightarrow \infty} 5^{m}(n r)^{p_{r} m}\left(p_{r} m+1\right)^{p_{r} m} \frac{1}{m^{\gamma_{r}\left(p_{r}-1\right) m}}$

$$
=\lim _{m \rightarrow \infty}\left(\frac{5^{1 / p_{r}} n r p_{r}^{\gamma_{r}\left(p_{r}-1\right) / p_{r}}}{\left(p_{r} m\right)^{\gamma_{r}\left(p_{r}-1\right) / p_{r}-1}}\right)^{p_{r} m}\left(\frac{p_{r} m+1}{p_{r} m}\right)^{p_{r} m}=0,
$$

$$
\begin{aligned}
& \lim _{m \rightarrow \infty} 5^{m+1 / p_{r}}(n r)^{p_{r} m+1}\left(p_{r} m+2\right)^{p_{r} m+1}\left(\frac{p_{r}+1}{p_{r} m+1}\right)^{\gamma_{r}\left(p_{r}-1\right)\left(m+1 / p_{r}\right)} \\
& =\lim _{m \rightarrow \infty}\left(\frac{5^{1 / p_{r}} n r\left(p_{r}+1\right)^{\gamma_{r}\left(p_{r}-1\right) / p_{r}}}{\left(p_{r} m+1\right)^{\gamma_{r}\left(p_{r}-1\right) / p_{r}-1}}\right)^{p_{r} m+1}\left(\frac{p_{r} m+2}{p_{r} m+1}\right)^{p_{r} m+1}=0 .
\end{aligned}
$$

With these limits at our disposal, we find that, for every $r \in \mathbb{N}$, there is $q_{r} \in \mathbb{N}$ such that, for every integer $m \geq q_{r}$ and $j \in\{1, \ldots, r-1\}$, we have the following auxiliary inequalities: 
(IV) $\quad(n r)^{p_{r} m+1}\left(p_{r} m+2\right)^{p_{r} m+1}<5^{-\left(m+1 / p_{r}\right)}\left(\frac{p_{r} m+1}{p_{r}+1}\right)^{\gamma_{r}\left(p_{r}-1\right)\left(m+1 / p_{r}\right)}$.

Step 3: the sets $G_{l}, Q_{r}, P_{r}, I_{r, j}$ and $J_{r, j}$. Being a $G_{\delta^{-}}$subset of $\mathbb{R}^{n}, G$ is equal to the intersection of a sequence $\left(G_{l}\right)_{l \in \mathbb{N}}$ of open subsets of $\mathbb{R}^{n}$ that we may suppose decreasing.

Proceeding as in the construction of the special compact cover of an open set, we find that each $G_{l}$ is the union of countably many compact cubes $Q_{l, m, h}$ that we may renumber as a sequence, say $\left(Q_{l, k}\right)_{k \in \mathbb{N}}$. Then for every $l, k \in \mathbb{N}$, we denote by $P_{l, k}$ the compact cube in $\mathbb{R}^{n}$ having the same center as $Q_{l, k}$ and $\frac{3}{2} \operatorname{diam}\left(Q_{l, k}\right)$ as diameter. Now we arrange $\mathbb{N}^{2}$ into a sequence $\left(\left(l_{r}, k_{r}\right)\right)_{r \in \mathbb{N}}$, set

$$
Q_{r}=Q_{l_{r}, k_{r}} \quad \text { and } \quad P_{r}=P_{l_{r}, k_{r}},
$$

and let $I_{r, j}$ and $J_{r, j}$ for $j \in\{1, \ldots, n\}$ be the compact intervals in $\mathbb{R}$ such that

$$
Q_{r}=\prod_{j=1}^{n} J_{r, j} \quad \text { and } \quad P_{r}=\prod_{j=1}^{n} I_{r, j} .
$$

Of course this construction leads to $J_{r, j} \subset I_{r, j}^{\mathrm{o}}$ for every $r \in \mathbb{N}$ and $j \in\{1, \ldots, n\}$.

Step 4: the functions $u_{r, j}$ and the numbers $m_{r}$. At this point, everything is set up to introduce the functions $u_{r, j}$ for $r \in \mathbb{N}$ and $j \in\{1, \ldots, n\}$, as well as the sequence $\left(m_{r}\right)_{r \in \mathbb{N}}$ of $\mathbb{N}$ by the following recursion.

An application of Proposition 5.5 to $\gamma=\gamma_{1}$ and $p=p_{1}$ leads to an integer $m_{1}>q_{1}$ and to functions $u_{1,1}, \ldots, u_{1, n} \in \mathrm{C}_{\infty}(\mathbb{R})$ such that, for every $j \in\{1, \ldots, n\}$,

(a) $u_{1, j}$ has no divergence point,

(b) $\operatorname{supp}\left(u_{1, j}\right) \subset I_{1, j}$,

(c) $\left\|u_{1, j}^{(k)}\right\|_{\mathbb{R}} \leq 2^{-m_{1}}, \forall k \in\left\{0,1, \ldots, m_{1}\right\}$,

(d) $\left\|u_{1, j}^{(k)}\right\|_{\mathbb{R}} \leq 2^{k} k^{\gamma_{1} k}, \forall k \in \mathbb{N}$,

(e) for every $t \in J_{1, j}$, one has either

$$
\left|u_{1, j}^{\left(p_{1} m_{1}\right)}(t)\right| \geq 5^{-m_{1}} m_{1}^{\gamma_{1}\left(p_{1}-1\right) m_{1}}
$$


or

$$
\left|u_{1, j}^{\left(p_{1} m_{1}+1\right)}(t)\right| \geq 5^{-\left(m_{1}+1 / p_{1}\right)}\left(\frac{p_{1} m_{1}+1}{p_{1}+1}\right)^{\gamma_{1}\left(p_{1}-1\right)\left(m_{1}+1 / p_{1}\right)} .
$$

Now, for an integer $r \geq 2$, if the functions $u_{t, j}$ for $t \in\{1, \ldots, r-1\}$ and $j \in\{1, \ldots, n\}$ and the integers $m_{1}, \ldots, m_{r-1}$ are obtained, we apply Proposition 5.5 to $\gamma=\gamma_{r}$ and $p=p_{r}$ and obtain an integer $m_{r}>$ $\sup \left\{p_{r-1} m_{r-1}, q_{r}\right\}$ and functions $u_{r, 1}, \ldots, u_{r, n} \in \mathrm{C}_{\infty}(\mathbb{R})$ such that, for every $j \in\{1, \ldots, n\}$,

(a) $u_{r, j}$ has no divergence point,

(b) $\operatorname{supp}\left(u_{r, j}\right) \subset I_{r, j}$,

(c) $\left\|u_{r, j}^{(k)}\right\|_{\mathbb{R}} \leq 2^{-m_{r}}, \forall k \in\left\{0,1, \ldots, m_{r}\right\}$,

(d) $\left\|u_{r, j}^{(k)}\right\|_{\mathbb{R}} \leq 2^{k} k^{\gamma_{r} k}, \forall k \in \mathbb{N}$,

(e) for every $t \in J_{r, j}$, one has either

$$
\left|u_{r, j}^{\left(p_{r} m_{r}\right)}(t)\right| \geq 5^{-m_{r}} m_{r}^{\gamma_{r}\left(p_{r}-1\right) m_{r}}
$$

or

$$
\left|u_{r, j}^{\left(p_{r} m_{r}+1\right)}(t)\right| \geq 5^{-\left(m_{r}+1 / p_{r}\right)}\left(\frac{p_{r} m_{r}+1}{p_{r}+1}\right)^{\gamma_{r}\left(p_{r}-1\right)\left(m_{r}+1 / p_{r}\right)} .
$$

Step 5: the functions $u_{r}$ and $u$. Finally, for every $r \in \mathbb{N}$, we define,

$$
u_{r}(x)=u_{r, 1}\left(x_{1}\right) \ldots u_{r, n}\left(x_{n}\right), \quad \forall x \in \mathbb{R}^{n},
$$

and consider the series $u=\sum_{r=1}^{\infty} u_{r}$. For every $k \in \mathbb{N}$, we certainly have $k \leq m_{k}$. Therefore, for every $\alpha \in \mathbb{N}_{0}^{n}$,

$$
\sum_{r=\sup \{1,|\alpha|\}}^{\infty}\left\|\mathrm{D}^{\alpha} u_{r}\right\|_{\mathbb{R}^{n}} \leq \sum_{r=\sup \{1,|\alpha|\}}^{\infty} 2^{-n m_{r}} \leq 1 ;
$$

this implies that $u$ is a bounded $\mathrm{C}_{\infty}$-function on $\mathbb{R}^{n}$. Moreover, for every $\alpha \in \mathbb{N}_{0}^{n}$ such that $|\alpha| \geq 1$, we have

$$
\begin{aligned}
\left\|\mathrm{D}^{\alpha} u\right\|_{\mathbb{R}^{n}} & \leq \sum_{r=1}^{|\alpha|-1}\left\|\mathrm{D}^{\alpha} u_{r}\right\|_{\mathbb{R}^{n}}+\sum_{r=|\alpha|}^{\infty}\left\|\mathrm{D}^{\alpha} u_{r}\right\|_{\mathbb{R}^{n}} \\
& \leq \sum_{r=1}^{|\alpha|-1} 2^{|\alpha|}|\alpha|^{\gamma|\alpha|}+1 \leq 3^{|\alpha|}|\alpha|^{\gamma|\alpha|},
\end{aligned}
$$

hence $u \in \Gamma_{\gamma}$.

To conclude, we prove that $G$ is the set of divergence points of $u$.

On the one hand, if $x \in \mathbb{R}^{n}$ does not belong to $G$, then $x \notin G_{l_{0}}$ for some $l_{0}$, hence $x \notin G_{l}$ for all $l \geq l_{0}$. This implies that $x$ belongs to an at most 
finite number of the $P_{r}$ 's. Therefore for $r$ large enough we have $\mathrm{D}^{\alpha} u_{r}(x)=0$ for every $\alpha \in \mathbb{N}_{0}^{n}$ and clearly $x$ is not a divergence point of $u$.

On the other hand, let us prove by contradiction that every element of $G$ is a divergence point of $u$. Suppose that $x \in G$ is not a divergence point of $u$. This implies the existence of $s \in \mathbb{N}$ such that

$$
\left|\mathrm{D}^{\beta} u(x)\right| \leq s^{|\beta|}|\beta|^{|\beta|} \quad \text { if }|\beta| \geq 1 .
$$

As $x$ belongs to each $G_{l}$, there is an integer $r>3 s$ such that $x \in Q_{r}$; in particular, $x_{j} \in J_{r, j}$ for every $j \in\{1, \ldots, n\}$.

Fix $j \in\{1, \ldots, n\}$. The consideration of the property (e) leads to the following two possibilities.

Case 1: We have

$$
\left|u_{r, j}^{\left(p_{r} m_{r}\right)}\left(x_{j}\right)\right| \geq 5^{-m_{r}} m_{r}^{\gamma_{r}\left(p_{r}-1\right) m_{r}} .
$$

Then we set $\alpha_{j}=p_{r} m_{r}$ and remark that

(1.i) the auxiliary inequality (III) leads to

$$
\left|u_{r, j}^{\left(\alpha_{j}\right)}\left(x_{j}\right)\right| \geq(n r)^{\alpha_{j}}\left(\alpha_{j}+1\right)^{\alpha_{j}},
$$

(1.ii) the use of $(\mathrm{I})$ in $(*)$ leads to

$$
\begin{aligned}
\sum_{t=1}^{r-1}\left|u_{t, j}^{\left(\alpha_{j}\right)}\left(x_{j}\right)\right| & +\sum_{t=r+1}^{\infty}\left|u_{t, j}^{\left(\alpha_{j}\right)}\left(x_{j}\right)\right| \\
\leq & \sum_{t=1}^{r-1} 2^{\alpha_{j}} \alpha_{j}^{\gamma_{t} \alpha_{j}}+\sum_{t=r+1}^{\infty} 2^{-m_{t}} \leq 2 \sum_{t=1}^{r-1} 2^{p_{r} m_{r}}\left(p_{r} m_{r}\right)^{\gamma_{t} p_{r} m_{r}} \\
& \leq \frac{1}{(*)} \sum_{t=1}^{r-1} 2^{-p_{r} m_{r}} 5^{-m_{r}} m_{r}^{\gamma_{r}\left(p_{r}-1\right) m_{r}} \leq \frac{1}{2}\left|u_{r, j}^{\left(\alpha_{j}\right)}\left(x_{j}\right)\right| .
\end{aligned}
$$

Case 2: (*) does not hold. Then we have

$$
\left|u_{r, j}^{\left(p_{r} m_{r}+1\right)}\left(x_{j}\right)\right| \geq 5^{-\left(m_{r}+1 / p_{r}\right)}\left(\frac{p_{r} m_{r}+1}{p_{r}+1}\right)^{\gamma_{r}\left(p_{r}-1\right)\left(m_{r}+1 / p_{r}\right)},
$$

we set $\alpha_{j}=p_{r} m_{r}+1$ and remark that

(2.i) the auxiliary inequality (IV) leads to

$$
\left|u_{r, j}^{\left(\alpha_{j}\right)}\left(x_{j}\right)\right| \geq(n r)^{\alpha_{j}}\left(\alpha_{j}+1\right)^{\alpha_{j}},
$$

(2.ii) the use of (II) in (*) leads to 


$$
\begin{aligned}
& \sum_{t=1}^{r-1}\left|u_{t, j}^{\left(\alpha_{j}\right)}\left(x_{j}\right)\right|+\sum_{t=r+1}^{\infty}\left|u_{t, j}^{\left(\alpha_{j}\right)}\left(x_{j}\right)\right| \\
& \quad \leq \sum_{t=1}^{r-1} 2^{\alpha_{j}} \alpha_{j}^{\gamma_{t} \alpha_{j}}+\sum_{t=r+1}^{\infty} 2^{-m_{t}} \leq 2 \sum_{t=1}^{r-1} 2^{p_{r} m_{r}+1}\left(p_{r} m_{r}+1\right)^{\gamma_{t}\left(p_{r} m_{r}+1\right)} \\
& \quad \leq \frac{1}{2} \sum_{t=1}^{r-1} 2^{-\left(p_{r} m_{r}+1\right)} 5^{-\left(m_{r}+1 / p_{r}\right)}\left(\frac{p_{r} m_{r}+1}{p_{r}+1}\right)^{\gamma_{r}\left(p_{r}-1\right)\left(m_{r}+1 / p_{r}\right)} \\
& \quad \leq \frac{1}{2}\left|u_{r, j}^{\left(\alpha_{j}\right)}\left(x_{j}\right)\right| .
\end{aligned}
$$

So setting $\alpha=\left(\alpha_{1}, \ldots, \alpha_{n}\right)$ yields

$$
\begin{aligned}
\left|\mathrm{D}^{\alpha} u(x)\right| & \geq\left|\mathrm{D}^{\alpha} u_{r}(x)\right|-\sum_{t=1}^{r-1}\left|\mathrm{D}^{\alpha} u_{t}(x)\right|-\sum_{t=r+1}^{\infty}\left|\mathrm{D}^{\alpha} u_{t}(x)\right| \\
& \geq\left|\mathrm{D}^{\alpha} u_{r}(x)\right|-\prod_{j=1}^{n}\left(\sum_{t=1}^{r-1}\left|u_{t, j}^{\left(\alpha_{j}\right)}\left(x_{j}\right)\right|+\sum_{t=r+1}^{\infty}\left|u_{t, j}^{\left(\alpha_{j}\right)}\left(x_{j}\right)\right|\right) \\
& \geq\left|\mathrm{D}^{\alpha} u_{r}(x)\right|-\prod_{j=1}^{n} \frac{1}{2}\left|u_{r, j}^{\left(\alpha_{j}\right)}\left(x_{j}\right)\right| \geq \frac{1}{2}\left|\mathrm{D}^{\alpha} u_{r}(x)\right| .
\end{aligned}
$$

For every $j \in\{1, \ldots, n\}$, as $\alpha_{j}$ belongs to $\left\{m_{r} p_{r}, m_{r} p_{r}+1\right\}$, we certainly have $\alpha_{j}+1 \geq|\alpha| / n$. Therefore

$$
\begin{aligned}
\left|\mathrm{D}^{\alpha} u(x)\right| & \geq \frac{1}{2}\left|\mathrm{D}^{\alpha} u_{r}(x)\right|=\frac{1}{2} \prod_{j=1}^{n}\left|u_{r, j}^{\left(\alpha_{j}\right)}\left(x_{j}\right)\right| \\
& \geq \frac{1}{2} \prod_{j=1}^{n}(n r)^{\alpha_{j}}\left(\alpha_{j}+1\right)^{\alpha_{j}} \geq \frac{1}{2}(n r)^{|\alpha|}\left(\frac{|\alpha|}{n}\right)^{|\alpha|}=\frac{1}{2} r^{|\alpha|}|\alpha|^{|\alpha|}
\end{aligned}
$$

and finally, as we have chosen $r>3 s$, we arrive at the following contradiction:

$$
\left|\mathrm{D}^{\alpha} u(x)\right| \geq \frac{1}{2}(3 s)^{|\alpha|}|\alpha|^{|\alpha|}>s^{|\alpha|}|\alpha|^{|\alpha|} .
$$

7. Proof of Theorem 1.1. We first fix some $\zeta \in] 1, \gamma[$. We next apply Theorem 6.1 to get $u \in \Gamma_{\zeta}$ having $G$ as its set of divergence points. We then apply Theorem 5.2 to get $h \in \Gamma_{\gamma}\left(\mathbb{R}^{n} \backslash G^{-}\right)$which is analytic on $\mathbb{R}^{n} \backslash G^{-}$ and such that

$$
\left\|\mathrm{D}^{\alpha} u-\mathrm{D}^{\alpha} h\right\|_{\left(\mathbb{R}^{n} \backslash G^{-}\right) \backslash K_{s+1}} \leq \frac{1}{s} \quad \text { if }|\alpha| \leq s \text { and } s \geq 2
$$

(where of course $K_{s}$ is the sth compact set corresponding to the special 
compact cover of the open set $\mathbb{R}^{n} \backslash G^{-}$). So the function

$$
f: \mathbb{R}^{n} \rightarrow \mathbb{R}, \quad x \mapsto \begin{cases}u(x) & \text { if } x \in G^{-}, \\ h(x) & \text { if } x \in \mathbb{R}^{n} \backslash G^{-},\end{cases}
$$

belongs to $\Gamma_{\gamma}$, is analytic on $\mathbb{R}^{n} \backslash G^{-}$and has $G$ as its set of divergence points.

We now apply Corollary 5.3 to get $g \in \Gamma_{\gamma}$ which is analytic on $\Omega$, identically 0 on no connected component of $\Omega$ and flat on $\mathbb{R}^{n} \backslash \Omega$; in particular, $g$ has no divergence point.

To conclude one just has to check that the function $f+g$ suits our purpose: $f+g$ certainly belongs to $\Gamma_{\gamma}$, is analytic on $\Omega$ (since $\Omega \subset \mathbb{R}^{n} \backslash G^{-}$) and has $G$ as its set of divergence points. Moreover, no point $x$ of $F$ can be a divergence point (since $F$ and $G$ are disjoint), nor an analytic point (this would imply that $f+g$ is analytic on some open ball $b$ centered at $x$; this in turn implies that $b$ and $G$ are disjoint, so $f$ must be analytic on $b$; finally, $g$ is analytic hence flat on $b$, contrary to the fact that $x$ must belong to the boundary of $\Omega$ ).

\section{References}

[1] R. P. Boas, A theorem on analytic functions of a real variable, Bull. Amer. Math. Soc. 41 (1935), 233-236.

[2] S. Mandelbrojt, Séries entières et transformées de Fourier, Applications, Publ. Math. Soc. Japan 10, 1967.

[3] H. Salzmann and K. Zeller, Singularitäten unendlich oft differenzierbarer Funktionen, Math. Z. 62 (1955), 354-367.

[4] J. Siciak, Punkty regularne $i$ osobliwe funkcji klasy $C_{\infty}$ [Regular and singular points of $C_{\infty}$ functions], Zeszyty Nauk. Polit. Śląsk. Ser. Mat.-Fiz. 48 (853) (1986), 127-146 (in Polish).

[5] H. Whitney, Analytic extensions of differentiable functions defined in closed sets, Trans. Amer. Math. Soc. 36 (1934), 63-89.

[6] Z. Zahorski, Sur l'ensemble des points singuliers d'une fonction d'une variable réelle admettant les dérivées de tous les ordres, Fund. Math. 34 (1947), 183-245.

Institut de Mathématique

Université de Liège

15, avenue des Tilleuls

B-4000 Liège, Belgium
Facultad de Matemáticas

Universidad de Valencia

Dr. Moliner 50

E-46100 Burjasot (Valencia), Spain 